\title{
Refining the characterization of residual function in hypertrophic cardiomyopathy through remote segment 4D strain analysis
}

\author{
Alessandro Satriano ${ }^{1,2^{*}}$, Nita Guron ${ }^{1,2}$, Yoko Mikami ${ }^{1,2}$, Naeem Merchant $^{1,3}$, Carmen P Lydell ${ }^{1,3}$, \\ Andrew G Howarth ${ }^{1,2}$, Nowell M Fine ${ }^{2}$, James A White ${ }^{1,2}$, Bobak Heydari, ${ }^{1,2}$ \\ From 19th Annual SCMR Scientific Sessions \\ Los Angeles, CA, USA. 27-30 January 2016
}

\section{Background}

Quantitative assessment of late gadolinium enhancement (LGE) by cardiovascular magnetic resonance imaging (CMR) has been associated with an increased risk of sudden cardiac death (SCD) in patients with hypertrophic cardiomyopathy (HCM). However, patients with lesser degrees of LGE may still remain at high risk of adverse cardiac events due to the diffuse pathophysiology of HCM. Non-invasive characterization of the degree of biomechanical strain within non-enhanced myocardium may be a novel marker of disease in patients with HCM.

\section{Methods}

Forty-one consecutive patients with HCM and 40 healthy controls underwent CMR at 3T (Skyra, Siemens, Germany). 4D strain analysis was performed using GIUSEPPE, an in-house software that allows tracking of a 3D patientspecific ventricular mesh across the cardiac cycle relying on a $4 \mathrm{D}$ velocity field reconstructed from feature tracking of routinely acquired, long and short axis cine SSFP views. Segments with LGE (defined as $>6$ SD beyond remote myocardium) were excluded to define mean global principal, radial, circumferential, and longitudinal strain values. HCM patients were categorized into those with total volume of $\mathrm{LGE} \geq 15 \%$ or $<15 \%$.

\section{Results}

Mean age of the HCM patients was $54.6 \pm 12.5$ years $(36 \%$ female) with mean LVEF of $76.4 \pm 9.0 \%$. Mean percentage of global LGE was $5.5 \pm 9.7 \%$ for the total cohort. All measures of $4 \mathrm{D}$ strain were significantly lower within the non-

${ }^{1}$ Stephenson Cardiac Imaging Centre, Calgary, AB, Canada Full list of author information is available at the end of the article enhanced myocardium of HCM patients as compared with healthy controls (Figure 1). Furthermore, 4D principal, circumferential, and radial strains within nonenhanced myocardium were significantly reduced in those patients with $\geq 15 \%$ LGE, as compared to those with $<15 \%$ (Figure 1). Linear regression analysis revealed a significant association between the degree of $4 \mathrm{D}$ principal strain within the non-enhanced myocardium of HCM patients and both volume of myocardial scar and mean myocardial wall thickness (Figure 2).

\section{Conclusions}

This study demonstrated significantly reduced 4D strain amplitude within non-enhanced segments of HCM patients as compared to normal controls. Furthermore, those patients with greater degrees of global LGE ( $\geq 15 \%)$ had significantly reduced parameters of $4 \mathrm{D}$ strain. Further studies using this technique for the assessment of regional 4D biomechanical strain to prognosticate sudden cardiac death and congestive heart failure within HCM patients are warranted.

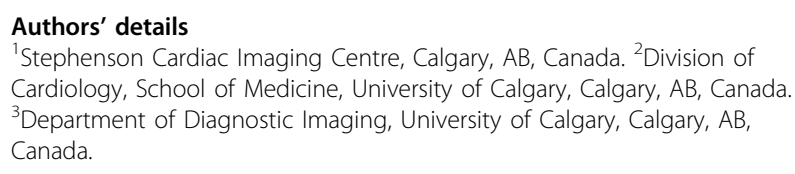

Published: 27 January 2016

doi:10.1186/1532-429X-18-S1-P234

Cite this article as: Satriano et al:: Refining the characterization of residual function in hypertrophic cardiomyopathy through remote segment 4D strain analysis. Journal of Cardiovascular Magnetic Resonance 2016 18(Suppl 1):P234. 


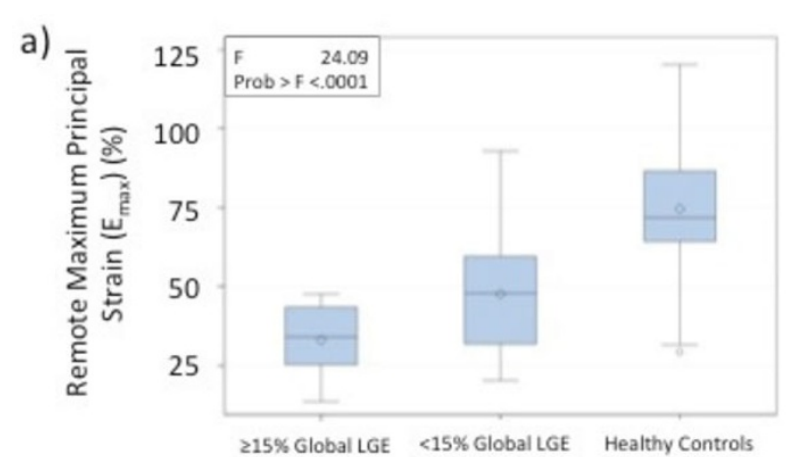

c)

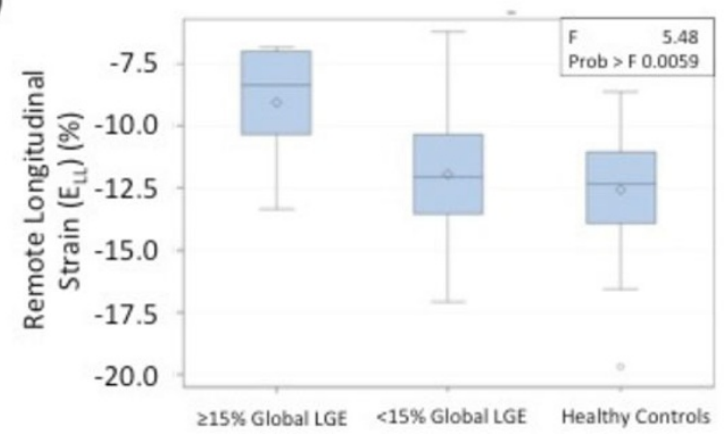

b)

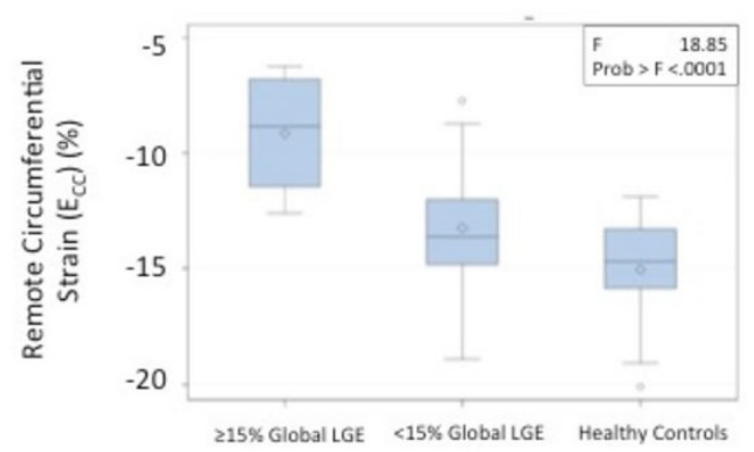

d)

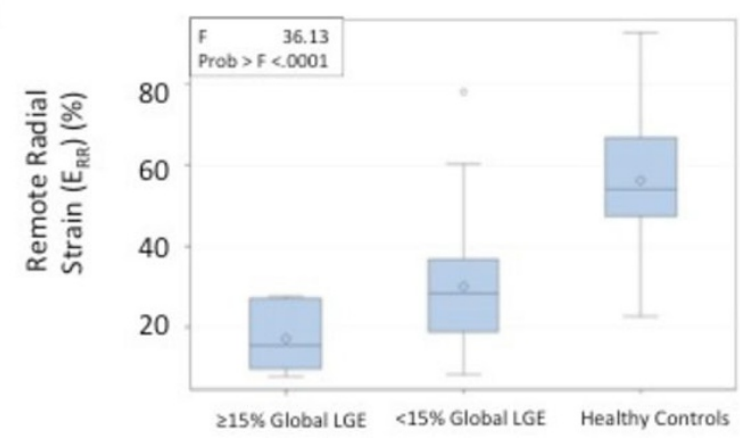

Figure 1 Boxplots representing the distributions of mean Maximum Principal (a), Circumferential (b), Longitudinal (c) and Radial (d) strain in remote tissue for HCM patients characterized by global LGE $>=15 \%,<15 \%$ and for healthy controls.

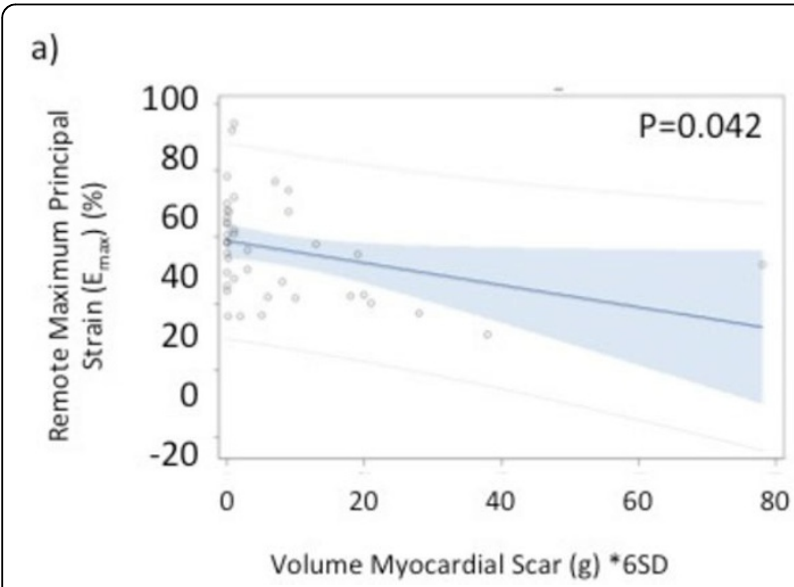

b)

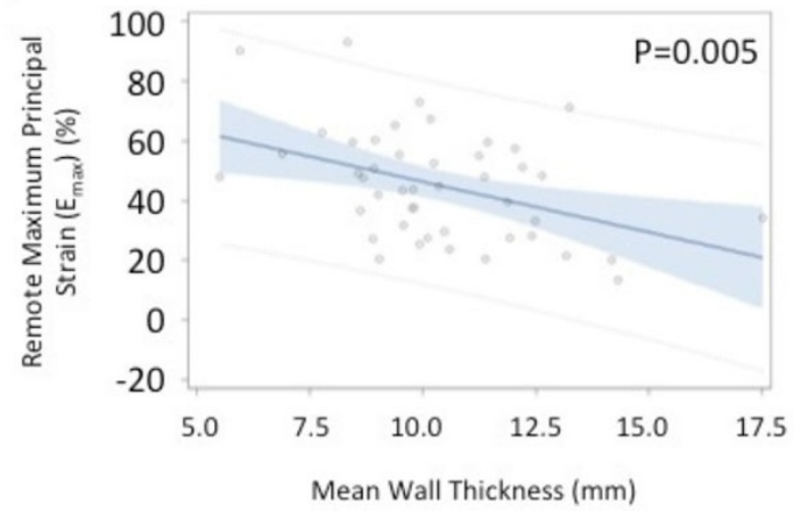

Figure 2 Linear regression of remote maximum principal strain vs. Volume of myocardial scar as estimated with a 6SD threshold (a) and vs. mean wall thickness (b). 